\title{
CURRENT STATUS OF HIV/AIDS-SYPHILIS CO-INFECTIONS: A RETROSPECTIVE MULTICENTRE STUDY
}

Figen Sarıgül', Murat Sayan², ${ }^{2}$, Dilara İnan ${ }^{4}$, Aydın Deveci', Nurgül Ceran ${ }^{6}$, Mustafa Kemal Çelen ${ }^{7}$, Atahan Çağatay ${ }^{8}$, Hülya Özkan Özdemir ${ }^{9}$, Ferit Kuşcu ${ }^{10}$, Gül Karagöz ${ }^{11}$, Yasemin Heper ${ }^{12}$, Oğuz Karabay ${ }^{13}$, Başak Dokuzoğuz ${ }^{14}$, Selçuk Kaya ${ }^{15}$, Nurettin Erben ${ }^{16}$, İlkay Karaoğlan ${ }^{17}$, Gülden Munis Ersöz ${ }^{18}$, Özgür Günal ${ }^{19}$, Çiğdem Hatipoğlu ${ }^{20}$, Selda Sayın Kutlu ${ }^{21}$, Ayhan Akbulut ${ }^{22}$, Rabin Saba ${ }^{23}$, Alper Şener ${ }^{24}$, Seyit Ali Büyüktuna ${ }^{25}$ ${ }^{1}$ Infectious Disease and Clinical Microbiology Department, Antalya and Research Hospital, Health Sciences University, Antalya, Turkey ${ }^{2}$ PCR Unit, Clinical Laboratory, Faculty of Medicine, Kocaeli University, Kocaeli, Turkey

${ }^{3}$ Research Centre of Experiment Health Sciences, Near East University, Nicosia, Northern Cyprus

${ }^{4}$ Infectious Disease and Clinical Microbiology Department, Faculty of Medicine, Akdeniz University, Antalya, Turkey ${ }^{5}$ Infectious Disease and Clinical Microbiology Department, Faculty of Medicine, Samsun 19 Mayıs University, Samsun, Turkey

${ }^{6}$ Infectious Disease and Clinical Microbiology Department, Istanbul Haydarpasa Numune Education and Research Hospital, Health Sciences University, Istanbul, Turkey

${ }^{7}$ Infectious Disease and Clinical Microbiology Department, Faculty of Medicine, Diyarbakir University, Diyarbakır, Turkey

${ }^{8}$ Infectious Disease and Clinical Microbiology Department, Faculty of Medicine, Istanbul University, Istanbul, Turkey

9Infectious Disease and Clinical Microbiology Department, Bozyaka Education and Research Hospital, Health Sciences University, Izmir, Turkey

${ }^{10}$ Infectious Disease and Clinical Microbiology Department, Faculty of Medicine, Cukurova University, Adana, Turkey

${ }^{11}$ Infectious Disease and Clinical Microbiology Department, Istanbul Umraniye Education and Research Hospital, Health Sciences University,

Istanbul, Turkey

${ }^{12}$ Infectious Disease and Clinical Microbiology Department, Faculty of Medicine, Bursa University, Bursa, Turkey

${ }^{13}$ Infectious Disease and Clinical Microbiology Department, Faculty of Medicine, Sakarya University, Sakarya, Turkey

${ }^{14}$ Infectious Disease and Clinical Microbiology Department, Ankara Numune Education and Research Hospital, Health Sciences University,

Ankara, Turkey

${ }^{15}$ Infectious Disease and Clinical Microbiology Department, Faculty of Medicine, Karadeniz Technical University, Trabzon, Turkey

${ }^{16}$ Infectious Disease and Clinical Microbiology Department, Faculty of Medicine, Eskisehir University, Eskisehir, Turkey

${ }^{17}$ Infectious Disease and Clinical Microbiology Department, Faculty of Medicine, Gaziantep University, Gaziantep, Turkey

${ }^{18}$ Infectious Disease and Clinical Microbiology Department, Faculty of Medicine, Mersin University, Mersin, Turkey

${ }^{19}$ Infectious Disease and Clinical Microbiology Department, Samsun Education and Research Hospital, Health Sciences University, Samsun, Turkey

${ }^{20 I n f e c t i o u s ~ D i s e a s e ~ a n d ~ C l i n i c a l ~ M i c r o b i o l o g y ~ D e p a r t m e n t, ~ A n k a r a ~ E d u c a t i o n ~ a n d ~ R e s e a r c h ~ H o s p i t a l, ~ H e a l t h ~ S c i e n c e s ~ U n i v e r s i t y, ~ A n k a r a, ~ T u r k e y ~}$

${ }^{21}$ Infectious Disease and Clinical Microbiology Department, Faculty of Medicine, Pamukkale University, Denizli, Turkey

${ }^{22}$ Infectious Disease and Clinical Microbiology Department, Faculty of Medicine, Firat University, Elazıg, Turkey

${ }^{23}$ Infectious Disease and Clinical Microbiology Department, Private Medstar Antalya Hospital, Antalya, Turkey

${ }^{24}$ Infectious Disease and Clinical Microbiology Department, Faculty of Medicine, Onsekiz Mart University, Canakkale, Turkey

${ }^{25}$ Infectious Disease and Clinical Microbiology Department, Faculty of Medicine, Cumhuriyet University, Sivas, Turkey

\section{SUMMARY}

Objective: Treponema pallidum and HIV are transmitted frequently through sexual contact, these agents with epidemiological similarities coinfect the same host. The current number of HIV-infected cases in Turkey is increasing. For this reason, we aimed to reveal the characteristics of syphilis in HIVIAIDS cases.

Methods: A retrospective longitudinal cohort study was performed, patients were followed up at 24 clinics in 16 cities from all seven regions of Turkey between January 2010 to April 2018. We examined the socio-demographic characteristics, laboratory parameters and neurosyphilis association in HIV/AIDS-syphilis co-infected cases.

Results: Among 3,641 patients with HIV-1 infection, 291 (8\%) patients were diagnosed with syphilis co-infection. Most patients were older than 25 years (92\%), $96 \%$ were males, $74 \%$ were working, $23 \%$ unemployed, and $3 \%$ were students. The three highest prevalence of syphilis were in Black Sea (10.3\%), Mediterranean ( $8.4 \%$ ) and Marmara Regions (7.4\%). As for sexual orientation, $46 \%$ were heterosexuals, $42 \%$ men who have sex with men (MSM), and no data available for $12 \%$. Patients with the number of $\mathrm{CD} 4+\leq 350 \mathrm{~mm}^{3}$ reached $46 \%, 17 \%$ of the patients received antiretroviral therapy and neurosyphilis association reached $9 \%$.

Conclusion: Although HIVIAIDS-syphilis co-infection status appeared high in heterosexuals, MSM had a moderate level increase in cases. Our results suggested syphilis co-infection in HIVIAIDS cases should be integral part of monitoring in a national sexual transmitted diseases surveillance system. However, our data may provide base for HIV/syphilis prevention and treatment efforts in the future.

Key words: syphilis, HIV, sexually transmitted diseases 
Address for correspondence: F. Sarigul, Health Science University, Antalya Education and Research Hospital, Department of Infectious Disease and Clinical Microbiology, 07050 Antalya, Turkey. E-mail: drfigensarigul@yahoo.com.tr

https://doi.org/10.21101/cejph.a5467

\section{INTRODUCTION}

Treponema pallidum and HIV are transmitted frequently through sexual contact, these agents with epidemiological similarities co-infect the same host. The relationship between the two diseases can be explained by behavioural factors as well as pathological mechanisms, such as facilitation of HIV transmission caused by genital ulceration and inflammation $(1,2)$. On the other hand, HIV/AIDS patients with syphilis have not only decreased CD4+ T lymphocyte but also increased plasma HIV RNA (3). Impaired humoral and cellular immunity depend on the strength of HIV infection and host defence against T. pallidum, leading to change in the clinical aspects and natural course of syphilis, increased number and syphilis lesion infectiousness, and shortened incubation time (4).

During the 1980s, a reduction in the incidence of sexually transmitted diseases (STDs) has occurred in many countries due to education and behavioural changes in response to the emergence of HIV/AIDS $(5,6)$. The rise in high-risk sexual practices and migration have contributed to the increased incidence of STDs since 2000 (7). Moreover, advances in epidemiological surveillance and patient detection methods have led to higher numbers of reported cases. In Western Europe, the reappearance of syphilis was mainly attributed to outbreaks among men who have sex with men (MSM) with novel sexual networks and high-risk sexual behaviours (8).

According to estimates of the World Health Organization (WHO), in 2014 syphilis seroprevalence in the key population among female sex workers and MSM was 2.3\% and 5.3\%, respectively, and each year 5.6 million new syphilis infections occur (9). Syphilis seroprevalence remains high in key populations especially in MSM and female sex workers worldwide, with at least one country in every WHO region reporting more than 5\% prevalence among female sex workers and MSM (9). Survey sampling often relies on nongovernmental organizations, working with hard-to-reach populations, resulting in studies that are limited to intervention areas. That is why there are limited WHO data about syphilis co-infection in HIV/AIDS patients.

In December 2017, the number of HIV-infected individuals in Turkey was 16.644 according to surveillance database of the Turkish Ministry of Health (MoH) (10). Heterosexual transmission has been the predominant mode of HIV acquisition in Turkey since 1985 and currently accounts for $35.4 \%$ of the estimated total people living with HIV and AIDS in Turkey in 2017 (10). Transmission among MSM was only $13.4 \%$, unknown transmission was high $-47.6 \%$, the others were nosocomial, among intravenous drug users, and mother to child transmission. However, recently published developed mathematical model showed that official and HIV drug data from intercontinental marketing service data, starting from 1994, was poor in Turkey; the predicted HIV cases continue to increase rapidly and are far greater than the exact cases (11).
According to $\mathrm{MoH}$ data, it is impossible to know the prevalence of HIV/syphilis co-infection, since there is no co-infection data in the notification and investigation records of acquired syphilis with HIV/AIDS. To obtain this information, it would be necessary to establish relationships between databases referring to each of the diseases using nominal data, which are not publicly available. Thus, we can justify the investigation of the prevalence of HIV/ AIDS-syphilis co-infection in a specialized care services, in order to contribute to the proposal of prevention and control measures of these diseases. In Turkey, there is only one study on syphilis prevalence of HIV-1 infected cases performed in a state hospital in Istanbul, in which $T$. pallidum exposure was determined in $12.9 \%$ of HIV/AIDS patients (12).

Our study intends to determine the current status of syphilis and demonstrates the characteristics among HIV/AIDS patients across Turkey where accurate data are absent.

\section{MATERIALS AND METHODS}

In this retrospective longitudinal cohort study, patients (aged $\geq 18$ years) were followed up at 24 clinics in 16 cities of Turkey between January 2010 to April 2018. The clinics are located in seven regions of Turkey: Marmara, Black Sea, Eastern Anatolia, Southeastern Anatolia, Central Anatolia, Mediterranean, and Aegean Regions and the clinics are reference hospitals in their regions for HIV/AIDS patients. The diagnosis of HIV/AIDS and syphilis were based on clinical manifestation supported by laboratory examination.

All of the study patients' records in the $\mathrm{MoH}$ surveillance database were categorized as HIV-1 infected according to the European AIDS Clinical Society (EACS) Guideline (13). For the HIV infection staging was used the US Centers for Disease Control and Prevention (CDC) classification system (1). Diagnosis of syphilis and staging was also confirmed in accordance with CDC STD treatment guideline (1).

The records of HIV-syphilis co-infected patients were analyzed retrospectively, i.e., socio-demographic including age, gender, nationality, marital status, partner HIV status, education, number of sexual partners, probable transmission route of HIV and syphilis infections, years of HIV seropositivity, clinical stage of syphilis and HIV/AIDS, use of ART and laboratory parameters; CD4+ T lymphocyte count and HIV viral load, semi-quantitative venereal diseases research laboratory (VDRL), and T. pallidum hemagglutination assay (TPHA) results and neurosyphilis association. The CD4+ T lymphocyte count threshold was chosen 350 cells $/ \mathrm{mm}^{3}$. Late presentation was defined as persons who were presenting for care with a CD4 cell count below 350 cells/ $\mathrm{mm}^{3}$ or presenting with an AIDS-defining event, regardless of the CD4 cell count (14). Disease stage was determined based on history, clinical findings, and laboratory results. Neurosyphilis cases were defined in accordance with the International Union 
Table 1. Demographic characteristics of patients with HIVI AIDS-syphilis and probable routes of transmission $(N=3,641)$

\begin{tabular}{|c|c|}
\hline Characteristic & Study group \\
\hline Patient, HIVIAIDS-syphilis, n (\%) & $291(8)$ of $3,641(100)$ \\
\hline \multicolumn{2}{|l|}{ Gender, n (\%) } \\
\hline Female & $13(4)$ \\
\hline Male & $278(96)$ \\
\hline Age, median years (range) & $41(18-90)$ \\
\hline \multicolumn{2}{|l|}{ Age, $n(\%)$} \\
\hline $18-24$ & $22(8)$ \\
\hline $25-34$ & $86(29)$ \\
\hline $35-44$ & $90(31)$ \\
\hline $45+$ & $93(32)$ \\
\hline \multicolumn{2}{|l|}{ Nationality, n (\%) } \\
\hline Turkish & $279(96)$ \\
\hline Other ${ }^{a}$ & $12(4)$ \\
\hline \multicolumn{2}{|l|}{ Socio-economic status, n (\%) } \\
\hline Employed & $214(74)$ \\
\hline Unemployed & $67(23)$ \\
\hline Student & $10(3)$ \\
\hline \multicolumn{2}{|l|}{ Education status, $n(\%)$} \\
\hline Illiterate & $3(1)$ \\
\hline Primary school & $91(31)$ \\
\hline Secondary school & $39(13)$ \\
\hline High school & $97(33)$ \\
\hline University & $63(22)$ \\
\hline \multicolumn{2}{|l|}{ Marital status, $\mathrm{n}(\%)$} \\
\hline Married & $106(36)$ \\
\hline Single & $179(62)$ \\
\hline Widowed & $6(2)$ \\
\hline \multicolumn{2}{|l|}{ Transmission route of HIV, $n(\%)$} \\
\hline Heterosexual contact & $135(46)$ \\
\hline $\mathrm{MSM}^{\mathrm{b}}$ contact & $120(42)$ \\
\hline Unknown & $36(12)$ \\
\hline \multicolumn{2}{|l|}{ Partner HIV status, n (\%) } \\
\hline Negative & $227(78)$ \\
\hline Positive & $31(10)$ \\
\hline Unknown & $33(12)$ \\
\hline \multicolumn{2}{|l|}{ Number of sexual partners, $\mathrm{n}(\%)$} \\
\hline Single & $28(10)$ \\
\hline Multiple & $250(86)$ \\
\hline Unknown & $13(4)$ \\
\hline \multicolumn{2}{|l|}{ Condom usage, $\mathrm{n}(\%)$} \\
\hline Yes & $22(8)$ \\
\hline No & $166(57)$ \\
\hline Unknown & $103(35)$ \\
\hline
\end{tabular}

aOther nationality - Macedonia ( $n=2)$, Turkmenistan $(n=2)$, Thailand $(n=1)$, Germany $(n=1)$, South Africa $(n=1)$, Kenya $(n=1)$, Brazil $(n=1)$, Egypt $(n=1)$, Russia $(n=1)$, Afghanistan $(n=1)$

${ }^{\mathrm{b}} \mathrm{MSM}$ - men who have sex with men
Against Sexually Transmitted Infections (IUSTI) guideline and the cerebrospinal fluid (CSF) serology (positive TPHA \pm VDRL tests) and increased number of mononuclear cells were used for their diagnosis (15).

Syphilis diagnosis: serological tests included the quantitative non-treponemal VDRL as screening and a positive test VDRL was confirmed with TPHA.

HIV diagnosis: plasma samples were tested for HIV antibodies using 4th generation anti-HIV1-2 Ab/Ag enzyme-linked immunoassay (ELISA) and positive results were tested once using the same ELISA assays (AxSym; Abbott Laboratories, IL, USA and Cobas E 601 Analyzer Roche Diagnostic- Mannheim, Germany). Positive results were confirmed by HIV1/2 Western blotting (DIA PRO, HIV-1 LIA, Diagnostic Bioprobes Srl, Italy).

Commercial real-time PCR assay was used for HIV-1 RNA detection and quantification: QIAsymphony/Rotor-Gene Q platforms by artus HIV-1 QS- RGQ kit (Qiagen GmBH, Hilden, Germany), COBAS Ampliprep/COBAS TaqMan platforms by HIV-1 test kit (Roche Molecular Systems, CA, USA) and Abbott M2000 SP/Abbott Real Time platforms by HIV-1 test kit (Abbott Molecular Inc. IL, USA).

The Ethical Committee for Research at the Health Sciences University, Antalya, and Research Hospital approved the study protocol (Registration No: 201813/10).

\section{RESULTS}

A total of 3,641 patients with HIV/AIDS infection were followed, and 291 (8\%) patients were diagnosed with syphilis co-infection (Table 1). Male patients had a higher prevalence of having HIV/AIDS with syphilis (278 patients, 96\%) compared to female patients (13 patients, 4\%). The vast majority of patients with HIV/AIDS-syphilis co-infected were older than 25 years $(92 \%)$, mean age 41 years with the youngest 18 years old and the oldest 90 years old.

Possible route of syphilis transmission: 135/291 (46\%) occurred via heterosexual contact, 120/291 (42\%) among MSM, while $36 / 291(12 \%)$ had no data (most of them did not to want to reveal their sexual orientation or the transmission route was not found despite the detailed inquiry). Most of them were Turkish patients $(96 \%)$. The distribution of patients co-infected with HIV/AIDS-syphilis from Black Sea, Mediterranean, Marmara, Central Anatolia, Southeastern Anatolia, Aegean, and Eastern Anatolia regions were $10.3 \%, 8.4 \%, 7.4 \%, 7.3 \%, 7.3 \%, 6.4 \%$, and $2.5 \%$, respectively (Figure 1). The province of Istanbul of Marmara region had 63 syphilis/754 HIV-AIDS patients (8.3\%) (data not shown).

Based on the distribution of socioeconomic status, most of patients were unemployed and students. Most patients were single. The predominant number of patients had multiple seronegative. Most patients were not using condom and the attained education of the patients was mostly high school.

The number of patients simultaneously diagnosed with both infections was higher. Most of them received antiretroviral therapy. In diagnosed HIV/syphilis co-infection HIV RNA, CD4+ T lymphocyte count, VDRL and TPHA levels were shown. Neurosyphilis was diagnosed in 25/291(9\%) patients, seven of them had uveitis, 15 of them had clinical symptoms and the 


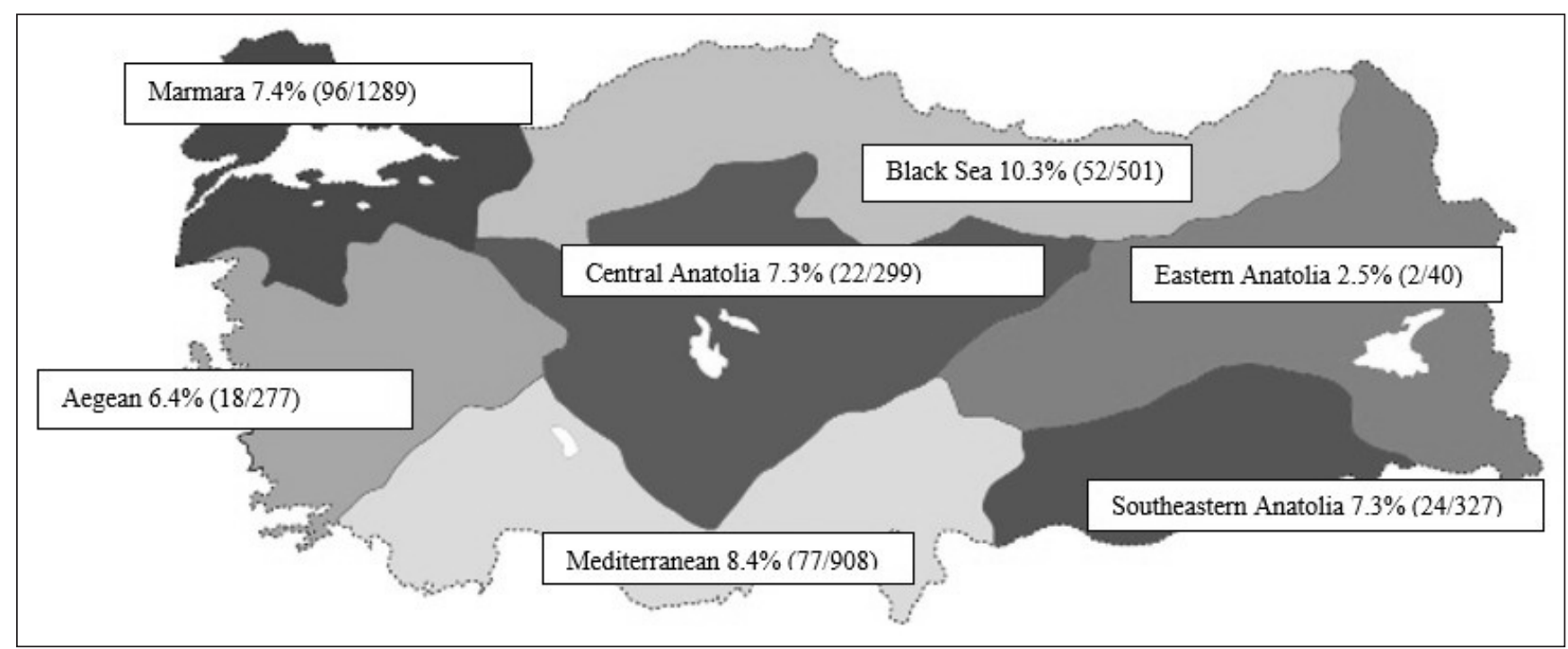

Fig. 1. Distribution of HIV/AIDS-syphilis patients in Turkey.

Table 2. Laboratory characteristics of patients with HIVIAIDSsyphilis $(N=3,641)$

\begin{tabular}{|c|c|}
\hline Characteristics & Study group \\
\hline Patient, HIVIAIDS-syphilis, n (\%) & $291(8)$ of $3,641(100)$ \\
\hline \multicolumn{2}{|l|}{ Duration of HIV + syphilis diagnosis, $n(\%)$} \\
\hline At the same time & $211(73)$ \\
\hline $1-2$ years & $26(9)$ \\
\hline $3-4$ years & $25(8)$ \\
\hline$>4$ years & $29(10)$ \\
\hline \multicolumn{2}{|l|}{ Antiretroviral drugs usage, $\mathrm{n}(\%)$} \\
\hline Yes & $50(17)$ \\
\hline No & $241(83)$ \\
\hline \multicolumn{2}{|l|}{ AIDS, $n(\%)$} \\
\hline Yes & $45(15)$ \\
\hline No & $246(85)$ \\
\hline Basal HIV-1 RNA, median copy/mL (range) & $9.7+\mathrm{E} 5(58-1.0+\mathrm{E} 9)$ \\
\hline $\begin{array}{l}\text { Basal CD4+ T lymphocyte count, median } \\
\text { cell/mm³ (range) }\end{array}$ & $384(5-1,520)$ \\
\hline \multicolumn{2}{|l|}{ Late presenter status, $\mathrm{n}(\%)$} \\
\hline$\leq 350$ cells $/ \mathrm{mm}^{3}$ & $134(46)$ \\
\hline$>350$ cells $/ \mathrm{mm}^{3}$ & $157(54)$ \\
\hline Serum VDRL, median titre (range) & $1 / 64(1 / 2-1,024)$ \\
\hline Serum TPHA, median titre (range) & $1 / 640(1 / 40-1 / 5,240)$ \\
\hline \multicolumn{2}{|l|}{ Syphilis stage, $n(\%)$} \\
\hline Primary & $30(10)$ \\
\hline Secondary & $65(22)$ \\
\hline Latent & $196(68)$ \\
\hline \multicolumn{2}{|l|}{ Neurosyphilis, $\mathrm{n}(\%)$} \\
\hline Yes & $25(9)$ \\
\hline No & $266(91)$ \\
\hline
\end{tabular}

others were asymptomatic. Three asymptomatic neurosyphilis had VDRL $\geq 1 / 32$ and CD4+ T lymphocyte count $\leq 350$ cells/ $\mathrm{mm}^{3}$. The laboratory characteristics of the patients are presented in Table 2.

\section{DISCUSSION}

According to the large-scale and long-term monitoring based on all regional levels of syphilis co-infection in HIV/AIDS cases reached $8 \%$ in Turkey. This data may be used as feedback to prevent and eradicate HIV/syphilis co-infection in the future. Syphilis seroprevalence in diagnosed HIV infected individuals was reported between $5.2-25 \%$ in other countries (16-18), although social, cultural and economic differences between countries may affect the frequency of STDs. Our study showed the magnitude and quality of HIV/AIDS-syphilis co-infection status for the first time in Turkey and revealed that syphilis co-infection prevalence in HIV/AIDS may be accepted as moderate level. On the other hand, the relationship between syphilis and HIV infection has not yet been fully understood. The study has proven that syphilis could increase the risk of HIV and vice versa, the spread of HIV infection might also increase the prevalence and incidence of syphilis (19). In the light of these ideas, our finding suggested that syphilis co-infection in HIV/AIDS cases should be integral part of monitoring in a national STDs surveillance system.

Our results showed that most of the patients were heterosexual (46\%), followed by MSM (42\%). According to 2017 Turkey official data, unknown transmission route in HIV/AIDS patients reached $47.6 \%(10)$. This very high ratio might be due to inability of the Turks to explain their sexual preferences more easily than other Europeans. Unfortunately, there is no data about transmission routes of not only syphilis but also HIV/AIDS-syphilis coinfected patients in official sources. However, our results were also different from a previous study done by Aydın et al., which showed that HIV/AIDS-syphilis co-infected patients reached $12.9 \%$ in Istanbul in a single centre, the transmission route was determined in 21.3\% of MSM (12). In 2000, 2013 and 2017, trans- 
mission of HIV/AIDS among heterosexual patients in Turkey was $50 \%, 46 \%, 35.4 \%$, and transmission among MSM was $8 \%, 9.9 \%$, $13.4 \%$, respectively (10). But recently published study showed that MSM transmission in HIV/AIDS patients had increased from $23 \%$ in 2012 to $43 \%$ in 2015 (20). The mathematical modelling also showed that the epidemic size of HIV infected-MSM cases in Marmara Region especially in Istanbul is higher than in other regions and cities (21). For this reason, an extraordinary increase of HIV/AIDS-syphilis co-infection can be expected in the near future among MSM individuals in the Marmara Region, especially in Istanbul. On the other hand, STD report from the USA CDC for the year 2017 indicate that syphilis prevalence is increasing among MSM (1-22). The reasons for increase in syphilis prevalence among HIV infected MSM are stated as multiple sexual partners, poor condom usage, finding HIV infected sexual partners on the web/smart phone applications, and common use of psychostimulants and drugs that improve sexual performance. In addition, there is a common belief that oral sex is "safer" regarding transmission of HIV infection, while syphilis may be transmitted through oral ulcer lesions (23).

Regarding the distribution of syphilis in HIV/AIDS patients by the regions, Black Sea Region had the highest prevalence (10.3\%). The second highest was in Mediterranean Region with $8.4 \%$, followed by Marmara Region with 7.4\%. The first two regions have the biggest number of tourism activity in Turkey*. Marmara Region has almost the largest Syrian and African refugee populations and it is next to Eastern Europe where the number of HIV and syphilis patients are increasing (24). On the other hand, Black Sea Region is adjacent to the Russian/Caucasian territory where the population of HIV and syphilis patients increased in 2017 as well (24). The relationship between these regions is tourism and commercial, so there is a lot of human traffic between these regions (25). Because of travellers' voluntary and involuntary sexual behaviour abroad, they are significant vectors especially of STDs' pathogens. The study, carried out in Turkey, reported that HIV-1 subtype A1 was increasing in Black Sea Region (26). HIV1-subtype B is known to be high in Turkey. The increase in subtype A1 indicates that there are too many entrances to the region from Caucasian countries where this type is more common (27). Our findings show that STDs prevention efforts may be more effective while focusing on different ways in every region.

The majority of HIV/AIDS patients who experienced syphilis were males (96\%) and older than 25 years (92\%) in our study. These results suggested that adult Turkish men might have more sexual activities, this might also lead to higher exposure to risk factors compared to women. For example, working far from their house/family or even in different cities might force them to visit prostitutes. Another factor that might cause a higher number of syphilis in male patients with HIV/AIDS was that some cases of syphilis in women were asymptomatic. Also, CDC reported that $2.6 \%$ of HIV patients were unemployed compared with $1 \%$ of employed (1), based on their socioeconomic status, most HIV/ AIDS patients with syphilis were working (74\%) in the study. In terms of marital status, more than half of our patients were single $(62 \%), 86 \%$ of our cases had multiple sexual partners. The study shows that the single ones had more multiple partners compared

*http://www.tuik.gov.tr/ to married people (23). Syphilis and HIV seroprevalence are increasing with increasing number of sexual partners. Males tend to behave differently and have highly risky attitudes to sexual activities than females. As for education attained, 97 (33\%) of our patients were high school graduates, 91 (31\%) of them were primary school graduates. The results of research in Istanbul by Aydin et al. also showed that half of their HIV infected patients $(50.7 \%)$ were primary school graduates, although no significant relationship has been found between education and syphilis seroprevalence (12). Correct use of latex condom is fairly effective for protection against HIV, syphilis and other STDs (28). However, the frequency of patients indicating routine condom use was low $(8 \%)$ and routine condom use was adopted mostly after being infected with HIV. In fact, almost $8 \%$ of our patients practice routine condom use, that may account for detecting a significant association between syphilis seroprevalence and condom usage. During casual sexual contact condom usage is infrequent in Turkey. In spite of the fact that registered prostitutes insist on the use of a condom, the customer unfortunately may not wish to use such prophylaxis. This is common among MSM population. In fact, pre-exposure prophylaxis (PrEP) was high among MSM in the United States (29), yet, individuals at-risk are not accessing PrEP because it is not in a state repayment plan in Turkey.

Our data confirm that both diseases facilitate the acquisition of the other, as $73 \%$ of syphilis patients, mostly in a latent stage (68\%), were diagnosed simultaneously with HIV infection. In spite of the late presenter patients (CD4 T cell count $\leq 350$ ) higher number $(46 \%)$, the fact that most patients were detected in symptom free and highly infective early latent stage, support the importance of regular syphilis laboratory check-ups in symptomless risk group of HIV/AIDS patients; 25/291 (9\%) patients were diagnosed with neurosyphilis. Except for three patients, all of them had neurological symptoms. Three asymptomatic neurosyphilis had VDRL $\geq 1 / 32$ and CD4+ T lymphocyte count $\leq 350$ cells $/ \mathrm{mm}^{3}$. The European Guidelines of the International IUSTI study period suggested that HIV infected patients may have increased risk of neurological involvement and CSF examination is recommended in all patients with $>1 / 32$ serum VDRL titre and $\leq 350$ cells $/ \mathrm{mm}^{3} \mathrm{CD} 4+\mathrm{T}$ cell lymphocyte count (15). However, CSF examination would not be recommended on the basis of high serum VDRL titre alone anymore (15). However, it should be kept in mind that neurosyphilis may be found at every stage of syphilis even if there are no neurological symptoms in patients as described above (30).

\section{CONCLUSION}

Turkey is located at the crossroads of Eastern Europe, Middle East and the Mediterranean basin. Increased mass movements and sex supply may cause an increase in STDs prevalence. Although HIV/AIDS-syphilis co-infection status appeared high in heterosexuals, MSM had a moderate level increase in cases in Turkey. It may be possible to force the limits due to the dynamics of late diagnosis. Our results suggested syphilis co-infection in HIV/AIDS cases should be an integral part of monitoring in a national STDs surveillance system. However, our data may provide base to HIV/syphilis prevention and treatment efforts in the future. 


\section{Acknowledgements}

We thank all patients who kindly gave consent to participate in this study.

\section{Conflict of Interests}

None declared

\section{REFERENCES}

1. Centers for Disease Control and Prevention. Sexually transmitted diseases (STDs). Data and statistics [Internet]. Atlanta: CDC [cited 2018 Apr 10]. Available from: https://www.cdc.gov/std/stats.

2. Global HIV Strategic Information Working Group. Biobehavioural survey guidelines for populations at risk for HIV. Geneva: WHO; 2017.

3. Kotsafti O, Paparizos V, Kourkounti S, Chatziioannou A, Nicolaidou E, Kapsimali V, et al. Early syphilis affects markers of HIV infection. Int J STD AIDS. 2016;27(9):739-45.

4. Scythes JB, Jones CM. Syphilis in the AIDS era: diagnostic dilemma and therapeutic challenge. Acta Microbiol Immunol Hung. 2013;60(2):93-116.

5. Zetola NM, Klausner JD. Syphilis and HIV Infection: an update. Clin Infect Dis. 2007;44(9):1222-8.

6. Savage EJ, Hughes G, Ison C, Lowndes CM; European Surveillance of Sexually Transmitted Infections network. Syphilis and gonorrhoea in men who have sex with men: a European overview. Euro Surveill. 2009 Nov 26;14(47). pii: 19417.

7. Abdulkader RS, Goswami K, Rai SK, Misra P, Kant S. HIV-risk behavior among the male migrant factory workers in a north Indian city. Indian J Community Med. 2015 Apr-Jun;40(2):108-15.

8. Heffelfinger JD, Swint EB, Berman SM, Weinstock HS. Trends in primary and secondary syphilis among men who have sex with men in the United States. Am J Public Health. 2007;97(6):1076-83.

9. World Health Organization. Global HIV/AIDS response [Internet]. [cited 2018 Apr 1]. Available from: http://www.who.int/hiv/data.

10. Turkish Ministry of Health. Distribution of HIV/AIDS patients by years in Turkey [Internet]. [cited 2017 Feb 11]. Available from: http://www. infeksiyondunyasi.org. (In Turkish.)

11. Sayan M, Hincal E, Sanlidag T, Kaymakamzade B, Sa'ad FT, Baba IA. Dynamics of HIV/AIDS in Turkey from 1985 to 2016. Qual Quant. 2018;52(Suppl 1):711-23.

12. Aydin OA, Karaosmanoğlu HK, Sayan M, Ince ER, Nazlıcan O. Seroprevalence and risk factors of syphilis among HIV/AIDS patients in Istanbul, Turkey. Cent Eur J Public Health. 2015;23(1):65-8.

13. European AIDS Clinical Society. Guidelines. Version 9.0 [Internet]. EACS; 2017 [cited Jul 25]. Avaialble from: http://www.eacsociety.org/ files/guidelines 9.0-english.pdf.

14. Limb M. More routine HIV testing is needed to reduce late diagnoses, says public health agency. BMJ. 2011 Nov 29;343:d7794. doi: 10.1136/ bmj.d7794.

15. Janier M, Hegyi V, Dupin N, Unemo M, Tiplica GS, Potočnik M, et al. 2014 European guideline on the management of syphilis [Internet]. [cited 2018 Apr 12]. Available from: https://www.iusti.org/regions/europe/pdf/ 2014/2014SyphilisguidelineEuropean.pdf.

16. Mata-Marín JA, Sandoval-Sánchez JJ, Huerta-García G, Arroyo-Anduiza CI, Alcalá-Martínez E, Mata-Marín LA, et al. Prevalence of antibodies against Treponema pallidum among HIV-positive patients in a tertiary care hospital in Mexico. Int J STD AIDS. 2015;26(2):81-5.
17. Badie BM, Yavari Z, Esmaeeli S, Paydary K, Emamzadeh-Fard S, Seyed Alinaghi SA, et al. Prevalence survey of infection with Treponema pal $\neg$ lidum among HIV-positive patients in Tehran. Asian Pac J Trop Biomed. 2013;3(4):334-6.

18. Tsachouridou O, Skoura L, Christaki E, Kollaras P, Sidiropoulou E, Zebekakis $\mathrm{P}$, et al. Syphilis on the rise: a prolonged syphilis outbreak among HIV- infected patients in Northern Greece. Germs. 2016;6(3):83-90.

19. Staneková $D$, Kramárová $P$, Wimmerová $S$, Hábeková $M$, Takáčová $M$, Mojzesová M. HIV and risk behaviour among men who have sex with Men in Slovakia (2008-2009). Cent Eur J Public Health. 2014;22(4):23944.

20. Sayan M, Sargin F, Inan D, Sevgi DY, Celikbas AK, Yasar K, et al. HIV-1 transmitted drug resistance mutations in newly diagnosed antiretroviralnaive patients in Turkey. AIDS Res Hum Retroviruses. 2016 Jan;32(1):2631 .

21. Sayan M. Turkey's HIV dynamics and the patient universe. In: HIV epidemiological status and prevention strategies in Turkey: KLIMIK HIV Study Group Meeting; 2017 Oct 14; Istanbul, Turkey [Internet]. [cited 2018 May 12]. Available from: https://www.klimik.org.tr. (In Turkish.)

22. European Centre for Disease Prevention and Control. Syphilis. Annual epidemiological report for 2016 [Internet]. Stockholm: ECDC; 2018 [cited 2018 Apr 19]. Available from: https://ecdc.europa.eu/en/syphilis.

23. Forsyth AD, Valdiserri RO. A state-level analysis of social and structural factors and HIV outcomes among men who have sex with men in the United States. AIDS Educ Prev. 2015;27(6):493-504.

24. Gokengin D, Oprea C, Begovac J, Horban A, Zeka AN, Sedlacek D, et al. HIV care in Central and Eastern Europe: How close are we to the target? Int J Infect Dis. 2018;70:121-30.

25. Napoli C, Dente MG, Kärki T, Riccardo F, Rossi P, Declich S; Network for the Control of Cross-Border Health Threats in the Mediterranean Basin and Black Sea. Screening for infectious diseases among newly arrived migrants: experiences and practices in non-EU countries of the Mediterranean Basin and Black Sea. Int J Environ Res Public Health. 2015 Dec 8;12(12):15550-8.

26. Sayan M, Sargýn F, Inan D, Sevgi DY, Celikbas AK, Yasar K, et al. Transmitted antiretroviral drug resistance mutations in newly diagnosed HIV-1 positive patients in Turkey. J Int AIDS Soc. 2014 Nov 2;17(4 Suppl 3):19750.

27. Fisgin NT, Sayan M, Deveci A, Gunal O, Kaya S. Molecular epidemiology of HIV-1 strains isolated from Black Sea Region of Turkey. In: XVIIIth Turkish Congress of Clinical Microbiology and Infectious Diseases; 2017 Mar 22-26; Antalya, Turkey. KLIMIK; 2017. p. 273. (In Turkish.)

28. Crosby RA, Charnigo R, Shrier LA. Prospective associations between perceived barriers to condom: use and "perfect use". Am J Prev Med 2014;47(1):70-2.

29. Cohen SE, Vittinghoff E, Bacon O, Doblecki-Lewis S, Postle BS, Feaster $\mathrm{DJ}$, et al. High interest in preexposure prophylaxis among men who have sex with men at risk for HIV infection: baseline data from the US PrEP Demonstration Project. J Acquir Immune Defic Syndr. 2015 Apr $1 ; 68(4): 439-48$.

30. Marra CM. Neurosyphilis. Continuum (Minneap Minn). 2015 Dec;21(6 Neuroinfectious Disease):1714-28 